\title{
Habilidades Socioemocionales en estudiantes que cursan una carrera universitaria en modalidad mixta
}

Dra. C. Ed. Claudia Angélica Sánchez Calderón. Mexicana

ORCID: https://orcid.org/0000-0002-7684-0247

casanchezc@uaemex.mx

Dr. Ed. José Luis Gama Vilchis. Mexicano

ORCID: http://orcid.org/0000-0003-3997-1413

jlgv@uaemex.mx

Dra. Ed. Irma Isabel Ortíz Valdéz. Mexicana

ORCID: http://orcid.org/0000-0002-0160-2797

iiortizv@uaemex.mx

Mtra. Ed. Amelia Guadalupe Sánchez Calderón. Mexicana

ORCID http://orcid.org/0000-0003-2716-7655

agsanchezc@uaemex.mx

Universidad Autónoma del Estado de México

Recibido: 31 de agosto del 2020

Aceptado: 10 de febrero del 2021

\section{Resumen}

La Organización de las Naciones Unidas para la Educación la Ciencia y la Cultura (UNESCO) América Latina y el Caribe, destaca la importancia de ampliar las oportunidades de acceso a la educación superior, sin ningún tipo de discriminación, buscando ampliar la cobertura mediante modalidades no escolares y mixtas, sin descuidar la calidad en la formación y la permanencia de los estudiantes. Las habilidades socioemocionales de los estudiantes representan un recurso personal para gestionar con eficacia el cambio personal, social y ambiental de forma realista y flexible, es decir, permiten afrontar las situaciones 
inmediatas, resolver problemas, tomar decisiones y mostrar optimismo, actitud positiva y automotivación, aspectos indispensables para el logro de la permanencia y el egreso en los estudios en modalidad mixta. El presente artículo tiene como propósito mostrar las habilidades socioemocionales con las que cuentan los estudiantes que cursan una licenciatura en modalidad mixta, en una universidad pública mexicana, reflexionando si éstas posibilitan su permanencia en los estudios. Se realizó una investigación de enfoque cuantitativo, de tipo exploratorio, con diseño de investigación no experimental, en la que participaron 303 estudiantes de la Licenciatura en Enfermería, que cursan la formación en modalidad mixta en una universidad pública mexicana. Se aplicó una escala de las habilidades socioemocionales que mide las dimensiones de Habilidades Intrapersonal e Interpersonal, Habilidades de Planificación, Habilidades para el Manejo del Estrés, así como las habilidades para mejorar sentimientos. Derivado de los resultados, se concluye que la dimensión predominante en los estudiantes de la carrera de Enfermería, son las Habilidades Intrapersonal e Interpersonal. Lo que nos indica que son capaces de reconocer y mostrar sus emociones de manera efectiva, mostrándose comprensivos con los demás para establecer relaciones interpersonales sanas y mantenerlas. Destacando la capacidad de autorregularse para mantener un equilibrio en su vida académica y profesional, lo que contribuye a permanecer y concluir los estudios universitarios.

Palabras clave: Habilidades Socioemocionales, modalidad mixta, autorregulación.

\title{
Social-emotional skills in students who are studying in a mixed mode university career
}

\begin{abstract}
The United Nations Educational, Scientific and Cultural Organization (UNESCO) Latin America and the Caribbean stresses the importance of expanding opportunities for access to higher education, without any kind of discrimination, seeking to expand coverage through non-school and mixed modalities, without neglecting the quality of training and retention of students. The social-emotional skills of the students represent a personal resource to effectively manage personal, social and environmental change in a realistic and flexible way, i.e., they allow them to face immediate situations, solve problems, make decisions and


show optimism, positive attitude and self-motivation, all of which are indispensable to achieve permanence and graduation in mixed modalities. The purpose of this article is to show the social-emotional skills that students who are studying a mixed mode degree in a Mexican public university have, reflecting on whether these skills make it possible for them to stay in their studies. It was carried out from a quantitative approach research, exploratory type, with non-experimental research design in which 303 students of the Bachelor of Nursing who are studying in mixed modality in a Mexican public university participated. A scale of social-emotional skills was applied, which measures the dimensions of Intrapersonal and Interpersonal Skills, Planning Skills, Stress Management Skills, as well as the skills to massage feelings. Derived from the results, we conclude that the predominant dimension in the students of the Nursing career is the Intrapersonal and Interpersonal Skills. This indicates that they can recognize and show their emotions in an effective way, showing understanding to others in order to establish healthy interpersonal relationships and maintain them; it highlights the capacity to self-regulate in order to maintain a balance in their academic and professional life, which contributes to staying and concluding their university studies.

Keywords: Social-emotional skills, mixed modality, self-regulation.

\section{Introducción}

Hoy en día la educación superior enfrenta innumerables desafíos como brindar educación de calidad, equitativa, eficiente con los recursos, lograr la vinculación institución-sociedad, renovar sus mecanismos de transparencia y rendición de cuentas, así como la ampliación de la cobertura, entre otros.

Una de las principales metas de las Instituciones de Educación Superior, se refiere a favorecer la permanencia y así, lograr altos índices de eficiencia terminal; diferentes estudios muestran que son la motivación, las capacidades de autorregulación y de autoconocimiento, pueden determinan la permanencia y conclusión exitosa de la trayectoria académica y formación universitaria. 


\section{Las Habilidades Sociales y Emocionales}

Para esta época de cambios es indispensable combinar la enseñanza de la ciencia y tecnología con las ciencias sociales y humanidad, ya que de acuerdo con Joseph Aoun (2017) la educación para el futuro:

"se concentrará en desarrollar las habilidades y competencias netamente humanas para las cuales, al menos por ahora, las máquinas no ofrecen una alternativa viable, tales como la empatía, el trato personal y las relaciones de grupo..." (ANUIES, 2018, p. 31).

De lo anterior, las diferentes habilidades de naturaleza humana y personal guardan importancia especial en la formación, lo que pone en un lado de la balanza los conocimientos científicos y tecnológicos, mientras que del otro lado, los aspectos sociales y humanos.

En este sentido, una forma de abonar a los aspectos antes señalados es promoviendo en los estudiantes las habilidades socioemocionales, que si bien cabe resaltar que la investigación en el tema de habilidades socioemocionales en el contexto escolar se encuentra en franco crecimiento, no es reciente si retomamos los dos componentes que el termino abarca.

Por un lado, la palabra emoción proviene del verbo latín emovere, mover, este implica todo sentimiento que mueve a la mente, hacia pensamientos, palabras y conductas, por lo que se dice que las emociones nos mueven y entintan cómo nos sentimos, pensamos y actuamos (AtentaMente, 2015).

Mientas que las habilidades sociales según Caballo (2005), son un conjunto de conductas que permiten al individuo desarrollarse en un contexto individual o interpersonal expresando sentimientos, actitudes, deseos, opiniones o derechos de un modo adecuado a la situación. (Lacunza A. y Contini N., 2011)

Al mencionar habilidades socioemocionales (HSE) es referirse a las herramientas que permiten a las personas entender y regular sus emociones, comprender las de los demás, sentir y mostrar empatía por los otros, establecer y desarrollar relaciones positivas, tomar 
decisiones responsables, así como definir y alcanzar metas personales (Hernández, Z. M, Trejo, T. Y \& Hernández, M., 2018).

Ser emocional y socialmente inteligente, de acuerdo con Bar-On, significa poder gestionar con eficacia el cambio personal, social y ambiental de forma realista y flexible, es decir, afrontar las situaciones inmediatas, resolver problemas, tomar decisiones y mostrar optimismo, actitud positiva y automotivación. Por lo que un clima social positivo se identifica por el desarrollo de un ambiente participativo y coherente, el cual permite y facilita una formación integral para el alumno, según señalan Molina y Pérez. Por lo que el clima social escolar es determinante para el éxito escolar (Fernández P, Cabello R. y Gutiérrez M., 2017).

Algunos estudios han generado evidencia de la importancia que tienen las habilidades socioemocionales para contribuir, entre otras cosas, a la permanencia de los estudios en el nivel profesional. En las últimas dos décadas se han hecho numerosas investigaciones analizando los beneficios que tiene la educación socioemocional en un contexto escolar. En torno a esto Durlak, Weissberg, Dymnicki, Taylor y Schellinger realizaron un metaanálisis de 213 programas de aprendizaje social y emocional donde observaron la existencia de diferencias significativas entre los grupos control y grupos experimentales en las competencias sociales y emocionales, actitudes, comportamiento y rendimiento académico. (Filella, Agulló, Pérez y Oriol, 2013).

Tradicionalmente las investigaciones se habían centrado con estudiantes de altas habilidades en su esfera cognitiva, dejando de lado otros aspectos no cognitivos relacionados con aspectos de la personalidad. En los últimos años, se han comenzado a incorporar distintas variables socioemocionales en el estudio de los estudiantes con altas habilidades, así como la percepción que tienen los agentes educativos sobre la competencia socioemocional de sus estudiantes

Según Altarejos, Calderón, Collell y Escudé (citado en Filella G., Agulló M., Pérez E. y Oriol G., 2013), en los centros escolares es fácil observar los efectos de la falta de competencia emocional en situaciones tales como comportamientos impulsivos, bloqueos a 
causa del estrés, baja autoestima, preocupación por situaciones familiares conflictivas, rechazo o maltrato entre compañeros, problemas de disciplina en las aulas o dificultades de integración de algunos estudiantes en el grupo clase. Así también autores como Abarca, Agullo, Carpena, LópezCassá, Obiols, Soldevila, Sotil, Escurra, Huerta, Rosas, Campos, y Llaños (citado en Filella G., Agulló M., Pérez E. y Oriol G., 2013), señalan que algunos de los estudios realizados hasta el momento en España, concluyen que los programas de educación socioemocional constituyen una excelente vía para contribuir a mejorar la capacidad adaptativa de sus receptores y, en consecuencia, optimizar su bienestar, mejorar su autorregulación y capacidad para gestionar los estados de ánimo negativos.

\section{Programas sobre Habilidades Socioemocionales}

Han existido distintas experiencias, programas, modelos, y estudios acerca de las habilidades socioemocionales en distintos países y por distintos autores e investigadores; donde se ha dado cuenta del impacto, por ejemplo la experiencia de implementación con tres programas de desarrollo socioemocional en escuelas de educación secundaria de Bután, México y Perú; el estudio concluye que es posible y deseable implementar este tipo de programas a gran escala y que resulta en mejoras significativas tanto en el bienestar de los estudiantes, como en el desempeño académico medido a través de los resultados de las pruebas estandarizadas nacionales. (Filella, Agulló, Pérez y Oriol, 2013).

Otra experiencia se refiere al programa Bienestar y Aprendizaje Socioemocional (BASE) se sustenta sobre los postulados teóricos del aprendizaje socioemocional y considera la evidencia acumulada sobre la efectividad de intervenciones tanto internacionales como en el contexto chileno (Marchant T., Milicic N., y Alamos P., 2013). La estructuración de áreas y actividades propuesta en el programa BASE se apoya en cuatro pilares conceptuales:

a) una perspectiva integral del desarrollo que considera como indisolubles lo cognitivo, lo afectivo, y lo ético.

b) un enfoque sistémicointeraccional en que los vínculos profesor-alumno, entre alumnos, y de cada estudiante consigo mismo, son considerados un aspecto central. 
c) la consideración de los procesos evolutivos de los estudiantes y la necesidad de que el contexto escolar favorezca la construcción de una narrativa positiva de sí mismo.

d) la importancia de la formación profesional docente que permita a los profesores atender las necesidades socioemocionales de los estudiantes.

Las áreas del programa se orientan a favorecer en los estudiantes el conocimiento de sí mismos, la valoración de las competencias personales, la comprensión acerca del efecto de las emociones en los propios comportamientos y los de los otros y la autorregulación. También se consideran como aspectos significativos dentro del programa la toma de perspectiva de los otros, las habilidades de comunicación, la relación con el mundo externo, la visión positiva del mundo y el afrontamiento pacífico de conflictos (Berger, C., Milicic, N., Alcalay, L., \& Torretti, A., 2011).

El más reciente programa llevado a cabo en México, el modelo Construye-t que puede tomar un papel de educación emocional, ya que de acuerdo a Bisquerra “... es una forma de prevención primaria inespecífica, consistente en intentar minimizar la vulnerabilidad a las disfunciones o prevenir su ocurrencia..." tomando como un desarrollo integral, ya que se les debe proporcionar los recursos y las estrategias adecuadas para enfrentar experiencias de la etapas de desarrollo, esto con el fin de fomentar actitudes positivas ante la vida como habilidades sociales y empatía, fungiendo como factores de desarrollo de bienestar personal y social. (Benetti, S., 2017).

Las habilidades socioemocionales que promueve, buscan que las personas comprendan sus emociones y puedan manejarlas, que establezcan y alcancen metas positivas, sentir y mostrar empatía hacia los otros, así como tomar decisiones responsables (SEP, 2015).

Esta propuesta de educación emocional consta de algunos componentes plasmados modelo propuesto por la Secretaría de Educación Pública (SEP) :

1. Dinámica de grupos: La habilidad esencial del líder implica iniciar y coordinar los esfuerzos de un grupo de gente. 
2. Negociar soluciones: Prevenir conflictos, solución de conflictos sociales y hacer de mediador son funciones esenciales en las relaciones interpersonales.

3. Conexión personal: Facilitan los encuentros de amigos, familiares, laborales, etc.

4. Análisis social: Ser capaz de detectar y tener una visión de los sentimientos, motivaciones y preocupaciones de los demás, ayuda a intimar y mantener buenas relaciones.

Con este programa que ha sido institucionalizado en México, se presume será una importante estrategia para el acompañamiento a los estudiantes, fortaleciendo sus capacidades y mejorando sus trayectorias escolares. Lo anterior, brevemente nos permite reconocer la importancia que tienen las habilidades socioemocionales para el desarrollo personal y escolar de todo individuo, cobrando especial valor en esta investigación, en el estudiante de la Licenciatura en Enfermería, donde la profesión le demanda habilidades específicas de autoconocimiento y relación social sana que le permita actuar con un espíritu de servicio acorde a su profesión.

Así, el objetivo de la investigación fue conocer las habilidades socioemocionales con las que cuentan los estudiantes que cursan la licenciatura en Enfermería, modalidad mixta en una universidad pública mexicana, y a partir de ello reflexionar si éstas favorecen su permanencia en los estudios.

\section{Metodología}

La educación superior en México vive innumerables problemáticas, sin embargo alternativas como cursar programas en modalidad mixta, han abierto las posibilidades para ampliar la cobertura a la formación profesional a otra parte de la población; por lo que resulta importante generar información en torno a las alternativas para lograr la permanencia de los estudiantes en esta modalidad; siendo una de ellas, mediante el desarrollo de las habilidades socioemocionales en los estudiantes. 
De lo anterior, surge el siguiente planteamiento: ¿Cuáles son las habilidades socioemocionales con las que cuentan los estudiantes de la Licenciatura en enfermería de una universidad pública mexicana, que cursan en modalidad mixta; y de qué manera favorecen la permanencia en los estudios?

Así el objetivo de la investigación se dirigió a conocer las habilidades socioemocionales con las que cuentan los estudiantes que cursan la licenciatura en Enfermería, modalidad mixta en una universidad pública mexicana, y a partir de ello reflexionar si éstas favorecen su permanencia en los estudios. La investigación se realizó desde el enfoque cuantitativo, de tipo exploratorio, transversal y no experimental.

Los participantes en la investigación fueron 303 estudiantes de la Licenciatura en Enfermería de una Universidad pública mexicana, distribuidos en 238 mujeres y 65 hombres con edad entre 18 y 35 años, identificados mediante un muestreo no probabilístico, con criterios de inclusión: ser estudiante en modalidad mixta, estudiante inscrito en la Licenciatura en Enfermería durante el periodo 2020A y pertenecer a la universidad pública seleccionada para desarrollar la investigación.

La recogida de datos de la Escala de Habilidades Socioemocionales de Sánchez C. y Col. (2018), se realizó asistiendo a la institución durante el desarrollo de las sesiones presenciales en los meses de febrero y marzo de 2020, y aplicado en formato de lápiz y papel. El instrumento se integra por 61 reactivos (Anexo), 15 de ellos miden las habilidades emocionales y 46 las habilidades sociales, se estructura en escala Likert, y se evocan a medir a la persona capaz de manejar, identificar y transmitir las Habilidades Socioemocionales, mediante las siguientes dimensiones: Habilidades Interpersonal e Intrapersonal, Habilidades para el manejo del Estrés, Habilidades para manejar sentimientos y Habilidades de Planificación.

El instrumento posee una varianza acumulada de 45.865, dividiéndose a su vez en varianzas por factor: $33.038 \%$ para el componente interpersonal e intrapersonal; $5.502 \%$ para el componente de habilidades de planificación; 4.655\% para manejo de estrés, y 2.670 para habilidades para manejar sentimientos. Finalmente, el instrumento cuenta con un Alpha de 
Cronbach de 0.95 en su primera validez. Los resultados se realizaron empleando estadística descriptiva congruente con el objetivo de la investigación.

\section{Resultados}

Derivado de la aplicación de la Escala de Habilidades Socioemocionales de Sánchez y Cols. (2018) a los 303 estudiantes de la licenciatura en Enfermería de una Universidad Pública Mexicana. A continuación se muestran los resultados obtenidos por dimensión que describe las Habilidades Interpersonal e Intrapersonal, Habilidades para el manejo del Estrés, Habilidades para manejar sentimientos, así como las Habilidades de Planificación.

Con relación a las Habilidades Intrapersonal e Interpersonal se encontró lo siguiente:

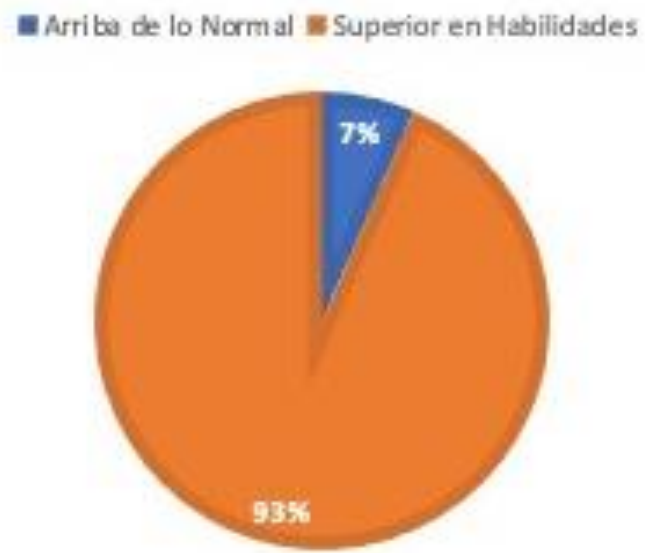

Figura 1. Habilidades Intrapersonal e Interpersonal

Como se observa en la figura 1, el 93\% del total de la muestra de la Licenciatura en Enfermería resultaron superiores en habilidades intrapersonal e interpersonal, lo cual nos indica que estas personas cuentan con alto grado de comprensión de las emociones de sí mismos, basta habilidad para ser asertivos y saben visualizarse de manera positiva. Así también, cuentan con alto sentido de autorrealización y de independencia.

De los 10 estudiantes que representaron el 7\% de la muestra, se ubicaron por arriba de lo normal en habilidades intrapersonal e interpersonal, lo que señala que cuentan con un 
alto grado de asertividad, comprensión hacia sí mismos, empáticos, independientes y con enorme capacidad de autovisualizarse positivamente.

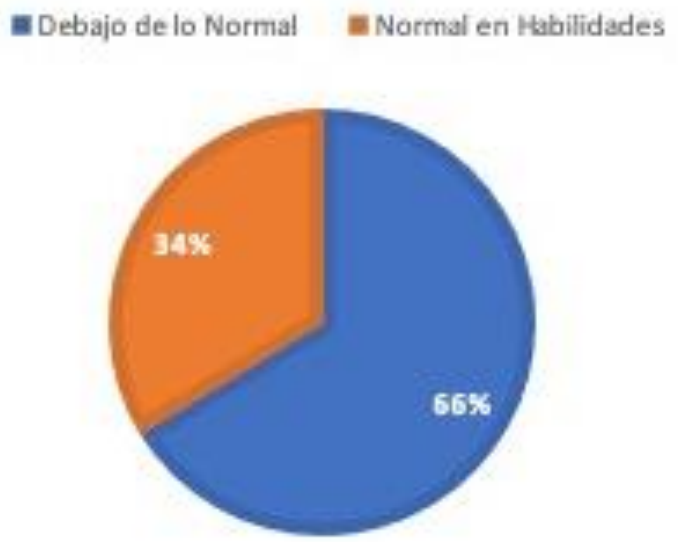

Figura 2. Habilidades de Planificación

Los 112 estudiantes que representan el 34\% de la muestra total de estudiantes de la Licenciatura en Enfermería, representado en la figura 2, se ubicaron como normal en habilidades de planificación y se caracterizan por mostrar capacidad para realizar planes y trazarse metas a corto, mediano y largo plazo con posibilidades de ser alcanzables y generalmente las logran, ya que son lo suficientemente metódicos y organizados. No pierden de vista su objetivo y se mantienen concentrados en lo que pretenden alcanzar, debido a que saben las habilidades que poseen y las ponen en práctica; así también logran resolver los conflictos que pueden surgir en el transcurso del logro de sus metas.

La otra parte, de la muestra conformada por 201 estudiantes y representado por el $66 \%$ del total. Se ubicaron por debajo de lo normal en habilidades de planificación, lo que nos indica que tienen marcadas dificultades para planificar su vida, establecer objetivos realizables, ya que son poco organizados y sistemáticos. Suelen perder de vista sus objetivos o los cambian de forma constante. Ante los conflictos se les dificulta saber que acciones tomar. 


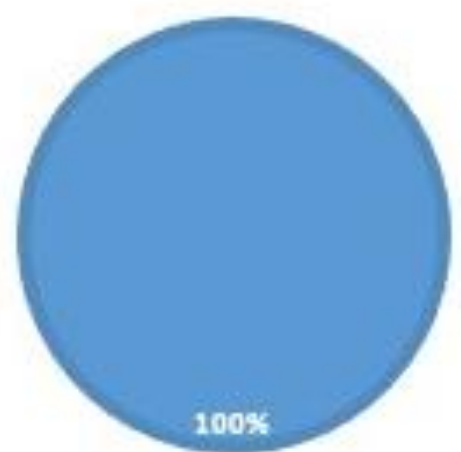

Figura 3. Habilidades para el Manejo del Estrés

Con relación a las habilidades para el manejo del estrés, mostrado en la figura 3, destaca que el total de la muestra obtuvo resultados deficientes, lo que nos indica que estas personas no cuentan con la capacidad de visualizar situaciones adversas de manera positiva, y por tanto suelen actuar de manera impulsiva. Ante el fracaso carecen de recursos resilientes para reaccionar y superarlo, o simplemente no pueden aceptar el fracaso.

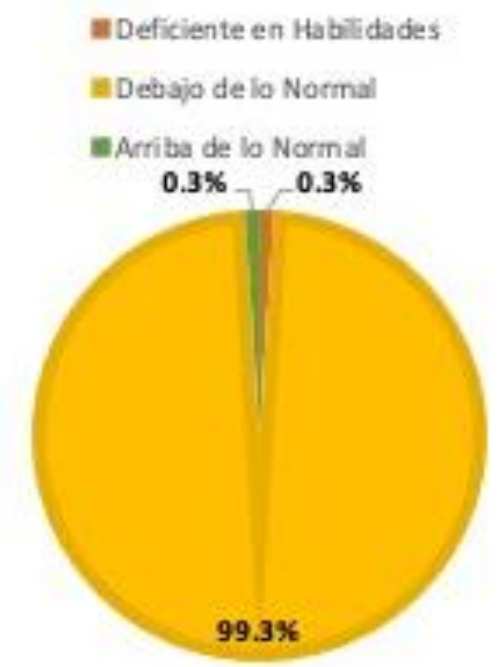

Figura 4. Habilidades para Manejar Sentimientos. 
Los resultados mostrados en la figura 4, destacan que 301 estudiantes que representan el $99.3 \%$ del total de la muestra de la Licenciatura en Enfermería, se ubican por debajo de lo normal; lo que nos indica una pronunciada dificultad para controlar sus emociones y sus miedos, se declaran poco empáticos y comprensivos; llegando a mostrarse indiferentes con los sentimientos de otros.

\section{Discusión}

A partir de los resultados que exploran las habilidades socioemocionales a una muestra de estudiantes de la licenciatura en enfermería, la dimensión de Habilidades Intrapersonal e Interpersonal, se muestran por arriba de la media, y considerando que son personas que se formaran para ejercer una profesión encaminada a la ayuda y la labor social, los resultados coinciden con lo señalado por Caballo, V. (2005) que destaca que las habilidades sociales, son un conjunto de conductas que permiten al individuo desarrollarse en un contexto individual o interpersonal expresando sentimientos, actitudes, deseos, opiniones o derechos de un modo adecuado a la situación. Generalmente, posibilitan la resolución de problemas inmediatos y la disminución de problemas futuros en la medida que el individuo respeta las conductas de los otros.

Así también, Bar-On, R. (1988) nos reafirma que los componentes Intrapersonal e Interpersonal, incluyen la comprensión emocional de sí mismo, asertividad, autoconcepto, autorrealización e independencia, al igual que la empatía, habilidad para establecer y mantener relaciones con otras personas y responsabilidad social. Todos ellos caracterizados por el perfil de egreso del profesional de la enfermería.

Así, la muestra estudiada revela elevadas capacidades Intrapersonales e Interpersonales, lo que nos indica que cuentan con una alta capacidad para reconocer y comprender sus emociones, al igual que saben expresarlas de una manera adecuada. Son capaces de comprender, aceptar y respetarse a sí mismos. Así también cuentan con habilidad para realizar lo que saben que pueden y disfrutan hacer, al igual que son independientes emocionalmente para tomar sus decisiones sin algún problema o dificultad. Son bastante 
capaces de comprender los sentimientos de los demás y respetar eso. Saben establecer y mantener una buena relación con las demás personas y son capaces de mostrarse como personas cooperativas y contribuyentes a un grupo social.

Bar-On, R. (1988) menciona que en las Habilidades Socioemocionales, dentro del componente interpersonal se encuentra el indicador de la independencia el cual describe como la capacidad para autodirigirse y ser capaz de visualizar sus necesidades y atenderlas. Por su parte Goleman (citado en Fragoso-Luzuriaga, R. 2015) nos señala que las Habilidades socioemocionales son un "conjunto de características clave para resolver con éxito los problemas vitales entre las que destacan: la habilidad de auto motivarse y persistir sobre las decepciones; controlar el impulso para demorar la gratificación; regular el humor; evitar trastornos que disminuyen las capacidades cognitivas; mostrar empatía, y generar esperanza”.

En los resultados de las habilidades de planificación se encontró que el $66 \%$ se clasificaron debajo de lo normal, lo que nos indica que estas personas se encuentran limitadas en su capacidad de planificar, así como de marcar objetivos alcanzables, ya que no son lo suficientemente organizadas, sistemáticas y metódicas. Suelen perder de vista sus objetivos o los cambian bastante seguido ya que puede que no tengan tan claro lo que se proponen. Pueden dejarse llevar por sus decepciones sin afrontarlas. Llegan a perder de vista sus necesidades y por tanto no las atienden, lo que también los lleva a no poder auto motivarse.

Así el 34\% que se encuentra dentro de la media en esta dimensión, destacan por su capacidad de autodirigirse y sentirse seguros de lo que hacen, siendo lo suficientemente metódicos para alcanzar sus objetivos, los visualizan y son alcanzables. Igualmente saben afrontar sus decepciones y persisten ante ellas. Muestran suficiente capacidad para visualizar sus necesidades y las atienden de una manera adecuada.

Respecto a la dimensión, Habilidades para el Manejo del Estrés, se encontró que el total de la muestra estudiada se clasificó muy por debajo de la media, considerándose muy deficiente, lo cual indica que estas personas carecen de las características que describe BarOn, R.(1988) en su componente del Manejo del Estrés, el cual "involucra la habilidad para mantenerse con una visión positiva frente a situaciones adversas, estresantes y fuertes 
emociones, enfrentando activa y positivamente el estrés; de igual manera involucra la habilidad para resistir o postergar un impulso para actuar y controlar nuestras emociones".

Por su parte, cuando se conjugan limitadas habilidades para el manejo del estrés con bajas habilidades de planificación, al momento en que la muestra se afronta a situaciones que sobrepasan su alcance, muestran muy poca o ninguna tolerancia a las situaciones estresantes, faltándoles la visión positiva ante esas situaciones y por lo tanto puede que lleguen a actuar de una manera impulsiva, sin dirección, y sin control de sus emociones.

Para la dimensión de Habilidades para Manejar Sentimientos se empata con el componente del estado de ánimo de Bar-On, R. (1988) el cual se trata de la "capacidad del individuo de ser consciente, comprender, controlar y expresar sus emociones de manera efectiva". También se trata de alcanzar la capacidad de la autoeficacia lo que significa que la persona es capaz de percibirse a sí misma y manejar el cómo realmente desea sentirse, muestra la habilidad para sentirse satisfecho con su vida, para disfrutar de sí mismo y de otros, y para divertirse y expresar sentimientos positivos.

De acuerdo con los resultados de la muestra estudiada el $100 \%$ se encuentra debajo de la media, lo que nos indica que a la mayoría se le dificulta reconocer y ser consciente de sus emociones, es poco probable que controlen y sepan expresar sus emociones de manera efectiva. Así mismo, no llegan a mostrar mucha habilidad para sentirse satisfechos con su vida, debido a su falta de control emocional. Estos resultados guardan relación con la dimensión de Habilidad del Manejo del Estrés, lo que nos indica congruencia del bajo promedio en ambas dimensiones.

En suma, la dimensión que predomina en los estudiantes de la carrera de Enfermería son las Habilidades Intrapersonal e Interpersonal. Lo que nos indica que son capaces de reconocer y mostrar sus emociones de manera efectiva, mostrándose comprensivos con los demás para establecer relaciones interpersonales sanas y mantenerlas. Destacando la capacidad de autorregularse para mantener un equilibrio en su vida académica y profesional, lo que contribuya a permanecer y concluir los estudios universitarios. 


\section{Conclusiones}

Las Habilidades Socioemocionales, en lo general se encuentran en la media en los estudiantes de la Licenciatura en Enfermería de una Universidad Pública Mexicana, lo que representa que en su mayoría cuentan con conocimiento y comprensión de sus emociones, basta habilidad para ser asertivos y saben visualizarse de manera positiva. Así también, cuentan con alto sentido de autorrealización y de independencia.

En términos generales muestran capacidad para realizar planes y trazarse metas a corto, mediano y largo plazo con posibilidades de ser alcanzables y generalmente las logran, ya que son lo suficientemente metódicos y organizados. No pierden de vista su objetivo y se mantienen concentrados en lo que pretenden alcanzar, debido a que saben las habilidades que poseen y las ponen en práctica; así también logran resolver los conflictos que pueden surgir en el transcurso del logro de sus metas.

Respecto al manejo del estrés, revelan la falta de capacidad para visualizar situaciones adversas de manera positiva, y por tanto suelen actuar de manera impulsiva. Ante el fracaso carecen de recursos resilientes para reaccionar y superarlo, o simplemente no pueden aceptar el fracaso. Con relación a las habilidades para manejar sentimientos, muestran una pronunciada dificultad para control sus emociones, para controlar sus miedos, se declaran poco empáticos y comprensivos; llegando a mostrarse indiferentes con los sentimientos de otros.

Finalmente, y considerando las características requeridas por un estudiante que cursa una profesión en modalidad mixta, y que refieren a contar con una alta capacidad de autoconocimiento, manejo de los emociones, planificación de sus tiempos, ser resiliente y tolerancia a la frustración; podemos decir que las habilidades socioemocionales que poseen los estudiantes la licenciatura en enfermería, son herramientas que pueden contribuir a permanecer y concluir los estudios universitarios de Licenciatura en Enfermería. 


\section{Referencias bibliográficas}

AtentaMente. (2015). Educar en Equilibrio: Las Habilidades Socioemocionales para el desarrollo personal y la mejora laboral del docente. Recuperado de: http://www.construyet.org.mx/resources/pdf/tpresenciales/Educar_en_Equilibrio.pd $\underline{\mathrm{f}}$

Asociación Nacional de Universidades e Instituciones de Educación Superior (2018) Visión y acción 2030. Propuesta de la ANUIES para renovar la educación superior en México. (1 ${ }^{\mathrm{a}}$ ed.) México, D. F. Publicación Editorial ANUIES. Disponible en http://www.anuies.mx/media/docs/avisos/pdf/VISION_Y_ACCION_2030.pdf

Bar-On, R. (1988). The development of a concept of psychological well-being, Unpublished doctoral dissertation, Rodhes University, South Africa. Recuperado de http://www.sciepub.com/reference/18498

Benetti, S. (3 marzo, 2017). Educación emocional. Bisquerra. Formación Integral Recuperado 10 diciembre 2020 http://formacion-integral.com.ar/website/?p=3365.

Berger, C., Milicic, N., Alcalay, L., \& Torretti, A. (2011), Programa para el Bienestar y Aprendizaje Socioemocional en estudiantes de tercero y cuarto grado: descripción y evaluación de impacto. Revista latinoamericana de Psicología Vol. 46 Issue 3 2014, pp.169-177. Recuperado de: https://www.sciencedirect.com/science/article/pii/S0120053414700202

Caballo, V. (2005). Manual de Evaluación y entrenamiento de las habilidades sociales. (6 ${ }^{\circ}$ Edición). Madrid: Siglo XXI.

Fernández P, Cabello R. y Gutiérrez M. (2017). Avances de la Investigación sobre competencias emocionales en educación. Revista Interuniversitaria de Formación del Profesorado, Núm. 88 (31.1), pp. 15-26. Recuperado de https://dialnet.unirioja.es/servlet/articulo?codigo=5980739

Filella G., Agulló M., Pérez E., Oriol G. (2013). Resultados de la aplicación de un programa de educación emocional en Educación Primaria. Barcelona. Ministerio de Ciencia e 
Innovación

Recuperado

de

https://www.unav.edu/publicaciones/revistas/index.php/estudios-sobreeducacion/article/viewFile/1864/1734

Fragoso-Luzuriaga, R. (2015) Inteligencia emocional y competencias emocionales en Educación Superior. ¿un mismo concepto?. Revista Iberoamericana de educación superior, 6 (6), 110-125. Recuperado el 10 de diciembre de 2020, de http://www.scielo.org.mx/scielo.php?script=sci_arttext\&pid=S2007$\underline{28722015000200006}$

Hernández, Z. M, Trejo, T. Y \& Hernández, M. (2018). El desarrollo de habilidades socioemocionales de los jóvenes en el contexto educativo. Instituto Nacional para la Evaluación de la Educación, México. Revista Red Recuperado de https://www.inee.edu.mx/images/stories/2018/Red9/10AlDia.pdf

Lacunza A. y Contini N. (2011) Las habilidades sociales en niños y adolescentes. Su importancia en la prevención de trastornos psicopatológicos. Fundamentos en Humanidades, vol. XII, núm. 23, 2011. Universidad Nacional de San Luis, Argentina

Marchant T., Milicic N., y Alamos P. (2013) Impacto en los niños de un programa de desarrollo Socio-emocional en dos colegios Vulnerables en Chile. Revista Iberoamericana de Evaluación Educativa, Vol 6, Núm. 2 pp. 167-186 Recuperado de http://www.rinace.net/riee/numeros/vol6-num2/art09_htm.html

Sánchez C y Cols. (2018). Instrumento de Habilidades Socioemocionales. UAEMéx, México.

SEP (2015) Programa Construye T. Disponible en https://www.gob.mx/sep/acciones-yprogramas/programa-construye-t-4598 


\section{ANEXO}

\section{"Escala de Habilidades Socioemocionales"}

Edad: __ Sexo: H M Estado Civil: Semestre:__ Licenciatura: procedencia:

Promedio general:

Trabajas: SI Actividad: NO

Esta escala pretende conocer las habilidades socioemocionales del alumno.

Por favor, marca con una "X" la casilla que se acerque a tu respuesta, siendo NUNCA (N) la carencia de las habilidades, CASI NUNCA $(\mathbf{C N})$ la aparición esporádica de las habilidades, ALGUNAS VECES (AV) la presencia periódicas de las habilidades, CASI SIEMPRE (CS) la presencia habitual de las habilidades y SIEMPRE (S) la presencia frecuente de las habilidades. Al responder ubica tu experiencia en los últimos tres meses.

Recuerda que la información que proporciones es confidencial y solo se usara con fines de investigación.

\begin{tabular}{|c|c|c|c|c|c|}
\hline \multirow{2}{*}{\multicolumn{6}{|c|}{$\begin{array}{l}\text { 1. Soy capaz de reconocer ante los demás que me encuentro incapacitado } \\
\text { emocionalmente para mis actividades cotidianas. }\end{array}$}} \\
\hline & & & & & \\
\hline \multicolumn{6}{|l|}{$\begin{array}{l}\text { 2. Me es fácil defender mis ideales de una manera sana, es decir sin agredir a los } \\
\text { demás. }\end{array}$} \\
\hline \multirow{2}{*}{\multicolumn{6}{|c|}{$\begin{array}{l}\text { 3. Acepto mis cualidades cuando alguien las menciona. } \\
\text { 4. Muestro entusiasmo al realizar mis actividades diarias expresando estar } \\
\text { satisfecho con lo que hago. }\end{array}$}} \\
\hline & & & & & \\
\hline \multicolumn{6}{|l|}{ 5. Me dejo persuadir fácilmente para tomar una decisión. } \\
\hline \multicolumn{6}{|l|}{ 6. Escucho con atención cuando me preguntan algo. } \\
\hline \multicolumn{6}{|l|}{ 7. Tiene relación las ideas que comparto con respecto al tema a tratar. } \\
\hline \multicolumn{6}{|l|}{ 8. Elijo la pregunta más importante para iniciar una conversación. } \\
\hline \multicolumn{6}{|l|}{ 9. Agradezco cuando es oportuno de forma amigable. } \\
\hline \multicolumn{6}{|l|}{ 10. Digo mi nombre al inicio de una presentación. } \\
\hline \multicolumn{6}{|l|}{ 11. Reconozco la importancia del esfuerzo de los demás de manera pública. } \\
\hline \multicolumn{6}{|l|}{$\begin{array}{l}\text { 12. Reconozco cómo se siente una persona por su tono de voz y por su expresión } \\
\text { corporal. }\end{array}$} \\
\hline \multicolumn{6}{|l|}{ 13. Me relaciono con los demás de forma respetuosa. } \\
\hline \multicolumn{6}{|l|}{ 14. Ayudo a resolver de forma adecuada las dudas que tengan otros. } \\
\hline \multicolumn{6}{|l|}{ 15. Pido ayuda cuando no puedo realizar algo. } \\
\hline \multicolumn{6}{|l|}{ 16. Me integro fácilmente a los grupos de trabajo. } \\
\hline \multicolumn{6}{|l|}{ 17. Explico a otros cómo se debe de realizar alguna actividad que domino. } \\
\hline \multicolumn{6}{|l|}{ 18. Reconozco mis propios errores ofreciendo disculpas. } \\
\hline \multicolumn{6}{|l|}{ 19. Puedo persuadir a un grupo de personas sin dificultad. } \\
\hline \multicolumn{6}{|l|}{$\begin{array}{l}\text { 20. Suelo ser paciente cuando se me presentan situaciones estresantes que no } \\
\text { puedo controlar fácilmente. }\end{array}$} \\
\hline \multicolumn{6}{|l|}{$\begin{array}{l}\text { 21. Me desespera cuando alguien no entiende lo que explico reaccionando de } \\
\text { manera agresiva. }\end{array}$} \\
\hline \multicolumn{6}{|l|}{$\begin{array}{l}\text { 22.Expreso a los otros los sentimientos que me provocan ciertas situaciones } \\
\text { cotidianas. }\end{array}$} \\
\hline \multicolumn{6}{|l|}{ 23. Muestro empatía cuando alguien me cuenta situaciones personales. } \\
\hline \multicolumn{6}{|l|}{ 24. Intento comprender las razones del enfado de la otra persona. } \\
\hline \multicolumn{6}{|l|}{ 25. Elijo la mejor manera de expresar mis afectos. } \\
\hline \multicolumn{6}{|l|}{ 26. Doy alternativas de solución en caso de que suceda lo que me causa miedo. } \\
\hline \multicolumn{6}{|l|}{ 27. Motivo a otros que se esfuercen más al realizar una actividad o tarea. } \\
\hline $\begin{array}{l}\text { 28. Muestro satisfacción y alegría expresando argumentos positivos que hagan } \\
\text { interesante la convivencia. }\end{array}$ & & & & & \\
\hline
\end{tabular}




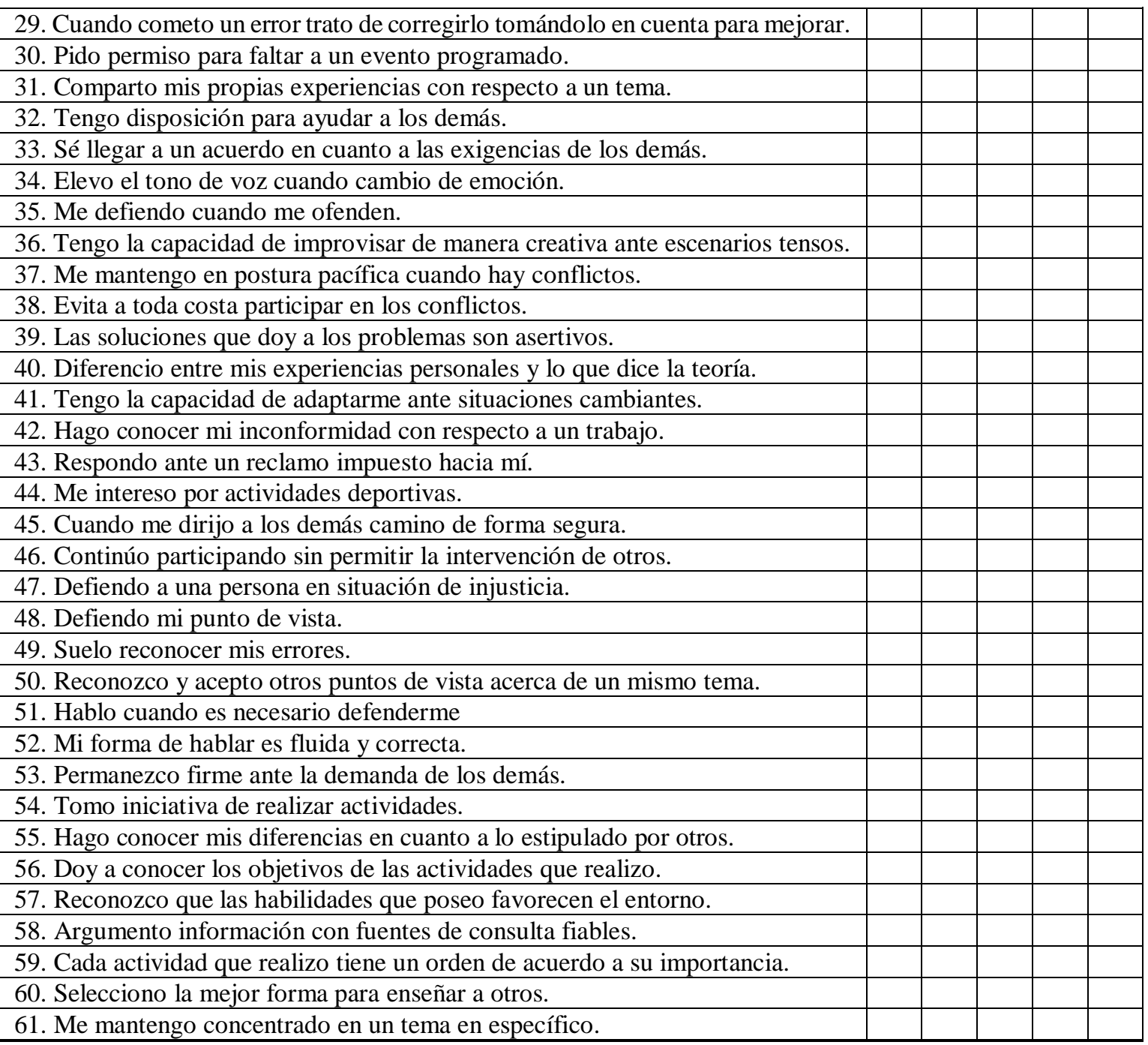

Gracias por su participación. 